\section{CRYSTALLOGRAPHIC COMMUNICATIONS}

ISSN 2056-9890

\section{Crystal structure of C-2-benzothiazole- $N$-methylnitrone}

\section{Roman Doroschuk}

Department of Inorganic Chemistry, Taras Shevchenko National University of Kyiv 64 Volodymyrska Str., 01033 Kyiv, Ukraine. *Correspondence e-mail: rdoroschuk@ukr.net

Received 23 June 2015; accepted 10 July 2015

Edited by C. Rizzoli, Universita degli Studi di Parma, Italy

The molecule of the title compound ssystematic name: $N$-[(benzothiazol-2-yl)methylidene]methylamine $\quad N$-oxide], $\mathrm{C}_{9} \mathrm{H}_{8} \mathrm{~N}_{2} \mathrm{OS}$, is close to planar [maximum deviation from the mean plane $=0.081(2) \AA]$, its conformation being stabilized by a strong intramolecular attractive $\mathrm{S}$...O interaction [2.6977 (16) $\AA$ ]. In the crystal, molecules are linked into centrosymmetric dimers by pairs of weak $\mathrm{C}-\mathrm{H}$. . O hydrogen bonds.

Keywords: crystal structure; benzothiazole; nitrone; S...O attractive interaction.

CCDC reference: 1411951

\section{Related literature}

For the 1,3-dipolar cycloaddition reaction of nitrones, see: Tufariello (1984); Torssell (1988). For the properties of benzothiazole derivatives, see: Bradshaw et al. (2002); Paramashivappa et al. (2003); Jimonet et al. (1999); Ul-Hasan et al. (2002); Şener et al. (2000); Mruthyunjayaswamy \& Shanthaveerappa (2000); Arpaci et al. (2002). For work by our group on nitrones, see: Doroschuk et al. (2006); Raspertova et al. (2002); Petkova et al. (2001). For attractive S-O interactions, see: Mokhir et al. (1999). For $\mathrm{N}-\mathrm{O}$ bond lengths in nitrones, see: Ruano et al. (2012). For van der Waals radii, see: Wells (1986). For the synthesis, see: Delpierre \& Lamchen (1965).

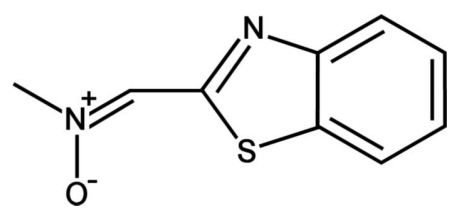

\section{Experimental}

2.1. Crystal data

$\mathrm{C}_{9} \mathrm{H}_{8} \mathrm{~N}_{2} \mathrm{OS}$

$M_{r}=192.23$

Triclinic, $P \overline{1}$

$a=5.5253$ (14) §

$b=7.4528(19) \AA$

$c=10.839$ (4) $\AA$

$\alpha=83.51(2)^{\circ}$

$\beta=85.79(3)^{\circ}$

\subsection{Data collection}

Oxford Diffraction Xcalibur 3 diffractometer

Absorption correction: multi-scan (CrysAlis PRO; Agilent, 2011) $T_{\min }=0.423, T_{\max }=0.994$

$\gamma=77.39(3)^{\circ}$

$V=432.2(2) \AA^{3}$

$Z=2$

Mo $K \alpha$ radiation

$\mu=0.33 \mathrm{~mm}^{-1}$

$T=294 \mathrm{~K}$

$0.4 \times 0.3 \times 0.2 \mathrm{~mm}$

\subsection{Refinement}

$R\left[F^{2}>2 \sigma\left(F^{2}\right)\right]=0.033$

$w R\left(F^{2}\right)=0.087$

$S=1.00$

1933 reflections

130 parameters

3491 measured reflections 1933 independent reflections 1477 reflections with $I>2 \sigma(I)$ $R_{\text {int }}=0.018$

Table 1

Hydrogen-bond geometry $\left(\AA,^{\circ}\right)$.

\begin{tabular}{lllll}
\hline$D-\mathrm{H} \cdots A$ & $D-\mathrm{H}$ & $\mathrm{H} \cdots A$ & $D \cdots A$ & $D-\mathrm{H} \cdots A$ \\
\hline $\mathrm{C} 5-\mathrm{H} 5 \cdots \mathrm{O} 1^{\mathrm{i}}$ & 0.93 & 2.53 & $3.331(2)$ & 145 \\
\hline
\end{tabular}

Symmetry code: (i) $-x+1,-y,-z+1$.

Data collection: CrysAlis PRO (Agilent, 2011); cell refinement: CrysAlis PRO; data reduction: CrysAlis PRO; program(s) used to solve structure: SHELXT (Sheldrick, 2015a); program(s) used to refine structure: SHELXL2014 (Sheldrick, 2015b); molecular graphics: OLEX2 (Dolomanov et al., 2009); software used to prepare material for publication: $O L E X 2$.

\section{Acknowledgements}

The author is grateful to the STC 'Institute for Syngle Crystals', 60 Lenina ave., Khar'kov 61001, Ukraine, for the singlecrystal X-ray diffraction data.

Supporting information for this paper is available from the IUCr electronic archives (Reference: RZ5162).

\section{References}

Agilent (2011). Crys Alis PRO. Agilent Technologies, Yarnton, England. Arpaci, Ö., Şener, E. A., Yalçin, I. \& Altanlar, N. (2002). Arch. Pharm. Pharm. Med. Chem. 335, 283-288.

Bradshaw, T. D., Chua, M. S., Browne, H. L., Trapani, V., Sausville, E. A. \& Stevens, M. F. G. (2002). Br. J. Cancer, 86, 1348-1354.

Delpierre, G. R. \& Lamchen, M. (1965). Q. Rev. Chem. Soc. 19, 329-348.

Dolomanov, O. V., Bourhis, L. J., Gildea, R. J., Howard, J. A. K. \& Puschmann, H. (2009). J. Appl. Cryst. 42, 339-341.

Doroschuk, R. A., Turov, A. V. \& Lampeka, R. D. (2006). Ukr. Khim. Zh. 72, 44-48.

Jimonet, P., Audiau, F., Barreau, M., Blanchard, J.-C., Boireau, A., Bour, Y., Coléno, M.-A., Doble, A., Doerflinger, G., Do Huu, C., Donat, M.-H., 


\section{data reports}

Duchesne, J. M., Ganil, P., Guérémy, C., Honoré, E., Just, B., Kerphirique, R., Gontier, S., Hubert, P., Laduron, P. M., Le Blevec, J., Meunier, M., Miquet, J., Nemecek, C., Pasquet, M., Piot, O., Pratt, J., Rataud, J., Reibaud, M., Stutzmann, J. \& Mignani, S. (1999). J. Med. Chem. 42, 2828-2843.

Ruano, J. L. G., Fraile, A., Núñez, A., Martín, M. R. \& Alonso, I. (2012). Heterocycles, 84, 913-928.

Mokhir, A. A., Domasevich, K. V., Kent Dalley, N., Kou, X., Gerasimchuk, N. N. \& Gerasimchuk, O. A. (1999). Inorg. Chim. Acta, 284, 85-98.

Mruthyunjayaswamy, B. H. M. \& Shanthaveerappa, B. K. (2000). Indian J. Chem. Sect. B, 39, 433-439.

Paramashivappa, R., Kumar, P. P., Rao, S. P. V. \& Rao, S. (2003). Bioorg. Med. Chem. Lett. 13, 657-660.

Petkova, E. G., Domasevitch, K. V., Gorichko, M. V., Zub, V. Y. \& Lampeka, R. D. (2001). Z. Naturforsch. B Chem. Sci. 56, 1264-1270.
Raspertova, I. V., Domasevich, K. V. \& Lampeka, R. D. (2002). Zh. Obshch. Khim. 72, 1854-1857.

Şener, E. A., Arpacı, Ö. T., Yalçın, İ. \& Altanlar, N. (2000). Farmaco, 55, $397-$ 405.

Sheldrick, G. M. (2015a). Acta Cryst. A71, 3-8.

Sheldrick, G. M. (2015b). Acta Cryst. C71, 3-8.

Torssell, K. G. (1988). Organic Synthesis, pp. 75-93. New York: VCH Publishers Inc.

Tufariello, J. J. (1984). 1,3-Dipolar Cycloaddition Chemistry, edited by A. Padwa, pp. 83-167. New York: John Wiley and Sons.

Ul-Hasan, M., Chohan, Z. H. \& Supuran, C. T. (2002). Main Group Met. Chem. 25, 291-296.

Wells, A. F. (1986). In Structural Inorganic Chemistry. Oxford: Clarendon Press. 


\section{supporting information}

Acta Cryst. (2015). E71, o578-0579 [https://doi.org/10.1107/S2056989015013262]

\section{Crystal structure of C-2-benzothiazole- $N$-methylnitrone}

\section{Roman Doroschuk}

\section{S1. Chemical context}

One of the most important approaches for the symthesis of various five-membered heterocyclic systems is the 1,3-dipolar cycloaddition reaction. The use as dipoles of compounds such as nitrile oxides and nitrones leads to a wide range of N,Ocontaining heterocyclic systems (isoxazole, isoxazoline, isoxazolidine; Tufariello, 1984), which have biological activity and can be used as starting materials for the synthesis of acyclic compounds (for example, 1,3-aminoalcohols by cleavage of the $\mathrm{N}-\mathrm{O}$ bond; Torssell, 1988). On the other hand, compounds containing the benzothiazole moiety are of biological and industrial interest. In fact, benzothiazole derivatives possess a wide spectrum of biological applications such as antitumor (Bradshaw et al., 2002), anti-inflammatory (Paramashivappa et al., 2003), anticonvulsant (Jimonet et al., 1999), and antimicrobial activities (Ul-Hasan et al., 2002; Şener et al., 2000; Mruthyunjayaswamy et al., 2000; Arpaci et al., 2002).

Following our studies on aromatic nitrone (Doroschuk et al., 2006; Raspertova et al., 2002; Petkova et al., 2001) in this paper we describe the structure of $C$-2-benzothiazole- $N$-methylnitrone.

\section{S2. Structural commentary}

In the molecule of the title compound (Fig. 1), the oxygen atom of the nitrone group exists in syn-conformation with respect to the sulfur atom of the benzothiazole moiety. This conformation is achieved due to a strong electrostatic intramolecular attractive $\mathrm{S} \cdots \mathrm{O}$ interaction. Thus, the $\mathrm{S} 1 \cdots \mathrm{O} 1$ distance $(2.6977(16) \AA)$ is significantly shorter than the sum of the van der Waals radii of $\mathrm{O}$ and $\mathrm{S}(1.5$ and $1.85 \AA$, respectively; Wells, 1986). A similar attractive $\mathrm{S} \cdots \mathrm{O}$ interaction is characteristic for molecules containing the thiazole moiety (Mokhir et al., 2002). The value of the $\mathrm{N} 1-\mathrm{C} 2$ bond length (1.298 (2) $\AA$ ) is typical for a $\mathrm{C}=\mathrm{N}$ bond (1.28 $\AA$; Wells, 1986), while the $\mathrm{N} 1-\mathrm{O} 1$ bond length (1.2763 (17) $\AA$ ) is slightly shorter than those usually observed for nitrone $\mathrm{N}-\mathrm{O}$ bonds (Petkova et al., 2001; Ruano et al., 2012). The heterocyclic ring system and the nitrone fragment $\mathrm{C} 1-\mathrm{N} 1-\mathrm{O} 1-\mathrm{C} 2$ are almost coplanar (the maximum deviation from the leastsquares mean plane is 0.081 (2) $\AA$ for atom C1), forming a dihedral angle of 3.40 (9) ${ }^{\circ}$. The bond lengths within the benzothiazole ring system are unexceptional.

\section{S3. Supramolecular features}

In the crystal (Fig. 2), centrosymmetrically-related molecules are linked into dimers via pairs of $\mathrm{C}-\mathrm{H} \cdots \mathrm{O}$ hydrogen bonds (Table 1).

\section{S4. Synthesis and crystallization}

$C$-2-Benzothiazole- $N$-methylnitrone was synthesized by condensation of the corresponding aldehyde with $\mathrm{N}$-methylhydroxylamine hydrochloride in the presence of base (Delpierre \& Lamchen, 1965).

2-Benzothiazolecarbaldehyde $(0.1632 \mathrm{~g}, 0.01 \mathrm{~mol}), \mathrm{CH}_{2} \mathrm{CL}_{2}(20 \mathrm{ml})$, and $\mathrm{N}$-metylhydroxylamine hydrochloride $(0.9175$ $\mathrm{g}, 0.011 \mathrm{~mol}$ ) were placed in a $50 \mathrm{~mL}$ flask, and the mixture was stirred at room temperature. To the resulting solution 
was added $\mathrm{NaHCO}_{3}(2.7675 \mathrm{~g}, 0.033 \mathrm{~mol})$. The reaction mixture was reluxed for $3 \mathrm{~h}$. The $\mathrm{NaCl}$ precipitate and excess of sodium bicarbonate was removed by filtration, and the filtrate was evaporated to give a solid (1.83g, yield 95\%). The solid was recrystallized from an absolute hexane solution.

\section{S5. Refinement}

One aromatic $\mathrm{H}$ atom (H6) could be located in a difference Fourier map and was refined freely. All other $\mathrm{H}$ atoms were placed geometrically and treated as riding, with $\mathrm{C}-\mathrm{H}=0.93-0.96 \AA$, and $U_{\text {iso }}(\mathrm{H})=1.5 U_{\text {eq }}(\mathrm{C})$ for methyl $\mathrm{H}$ atoms or $1.2 U_{\mathrm{eq}}(\mathrm{C})$ otherwise. A rotating model was used for the methyl $\mathrm{H}$ atoms. 36 outliers were omitted in the last cycles of refinement.

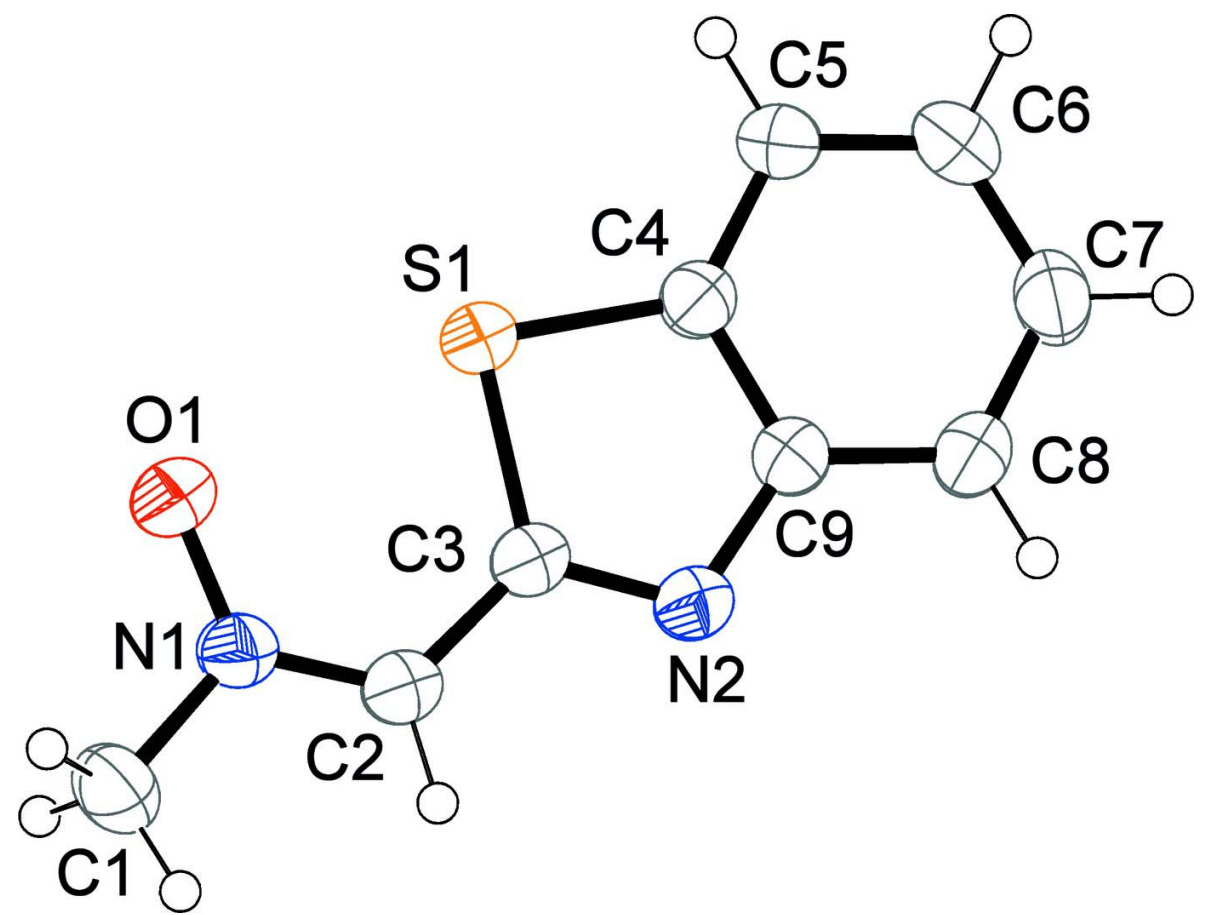

Figure 1

The molecular structure of the title compound with displacement ellipsoids drawn at the $50 \%$ probability level. 


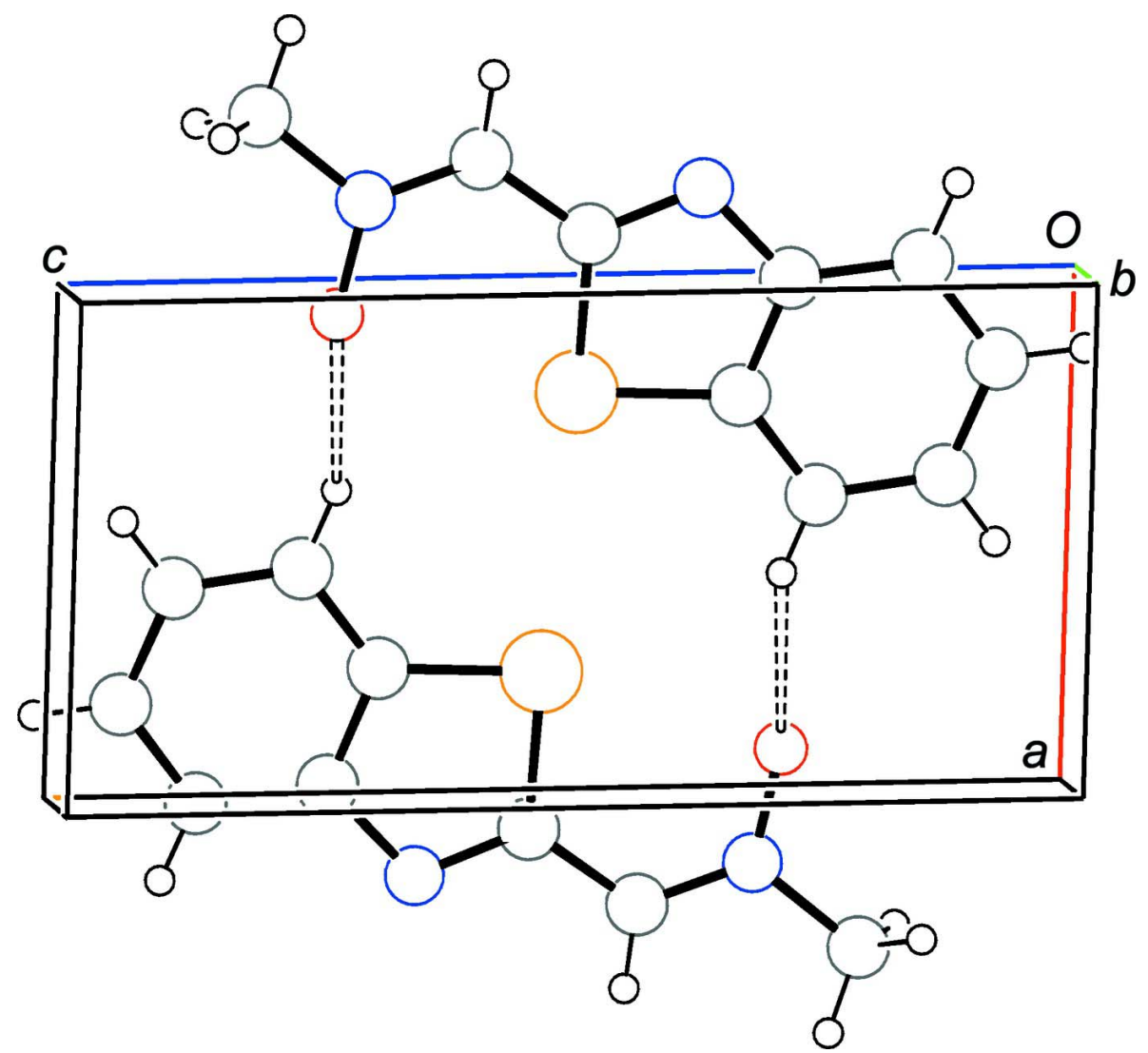

Figure 2

Partial crystal packing of the title compound approximately viewed along [ $\left[\begin{array}{lll}0 & 1 & 0\end{array}\right]$, showing the formation of a dimeric unit through a pair of $\mathrm{C}-\mathrm{H} \cdots \mathrm{O}$ hydrogen bonds (dashed lines).

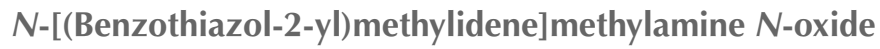

\section{Crystal data}

$\mathrm{C}_{9} \mathrm{H}_{8} \mathrm{~N}_{2} \mathrm{OS}$

$M_{r}=192.23$

Triclinic, $P \overline{1}$

$a=5.5253(14) \AA$

$b=7.4528(19) \AA$

$c=10.839(4) \AA$

$\alpha=83.51(2)^{\circ}$

$\beta=85.79(3)^{\circ}$

$\gamma=77.39(3)^{\circ}$

$V=432.2(2) \AA^{3}$

\section{Data collection}

Oxford Diffraction Xcalibur 3 diffractometer

Detector resolution: 16.1827 pixels $\mathrm{mm}^{-1}$

\section{$\omega$ scans}

Absorption correction: multi-scan

(CrysAlis PRO; Agilent, 2011)

$T_{\min }=0.423, T_{\max }=0.994$

3491 measured reflections
$Z=2$

$F(000)=200$

$D_{\mathrm{x}}=1.477 \mathrm{Mg} \mathrm{m}^{-3}$

Mo $K \alpha$ radiation, $\lambda=0.71073 \AA$

Cell parameters from 110 reflections

$\theta=18.2-8.2^{\circ}$

$\mu=0.33 \mathrm{~mm}^{-1}$

$T=294 \mathrm{~K}$

Block, colourless

$0.4 \times 0.3 \times 0.2 \mathrm{~mm}$

1933 independent reflections

1477 reflections with $I>2 \sigma(I)$

$R_{\text {int }}=0.018$

$\theta_{\text {max }}=27.5^{\circ}, \theta_{\min }=2.8^{\circ}$

$h=-7 \rightarrow 7$

$k=-9 \rightarrow 9$

$l=-14 \rightarrow 14$ 


\section{Refinement}

Refinement on $F^{2}$

Least-squares matrix: full

$R\left[F^{2}>2 \sigma\left(F^{2}\right)\right]=0.033$

$w R\left(F^{2}\right)=0.087$

$S=1.00$

1933 reflections

130 parameters

0 restraints

Primary atom site location: structure-invariant direct methods
Hydrogen site location: inferred from neighbouring sites

$\mathrm{H}$ atoms treated by a mixture of independent and constrained refinement

$w=1 /\left[\sigma^{2}\left(F_{\mathrm{o}}{ }^{2}\right)+(0.0521 P)^{2}\right]$ where $P=\left(F_{\mathrm{o}}{ }^{2}+2 F_{\mathrm{c}}{ }^{2}\right) / 3$

$(\Delta / \sigma)_{\max }<0.001$

$\Delta \rho_{\max }=0.24 \mathrm{e} \AA^{-3}$

$\Delta \rho_{\min }=-0.19 \mathrm{e} \AA^{-3}$

\section{Special details}

Geometry. All e.s.d.'s (except the e.s.d. in the dihedral angle between two 1.s. planes) are estimated using the full covariance matrix. The cell e.s.d.'s are taken into account individually in the estimation of e.s.d.'s in distances, angles and torsion angles; correlations between e.s.d.'s in cell parameters are only used when they are defined by crystal symmetry. An approximate (isotropic) treatment of cell e.s.d.'s is used for estimating e.s.d.'s involving 1.s. planes.

Fractional atomic coordinates and isotropic or equivalent isotropic displacement parameters $\left(\AA^{2}\right)$

\begin{tabular}{lllll}
\hline & $x$ & $y$ & $z$ & $U_{\text {iso }} * / U_{\text {eq }}$ \\
\hline O1 & $0.0619(2)$ & $0.21490(18)$ & $0.72954(11)$ & $0.0573(3)$ \\
S1 & $0.22145(7)$ & $0.17869(5)$ & $0.49058(4)$ & $0.04032(14)$ \\
N1 & $-0.1621(2)$ & $0.29247(17)$ & $0.70659(12)$ & $0.0406(3)$ \\
N2 & $-0.1774(2)$ & $0.33921(18)$ & $0.37522(12)$ & $0.0415(3)$ \\
C1 & $-0.3325(3)$ & $0.3346(3)$ & $0.81411(16)$ & $0.0536(4)$ \\
H1A & -0.2936 & 0.4351 & 0.8513 & $0.091(8)^{*}$ \\
H1B & -0.3159 & 0.2277 & 0.8738 & $0.078(7)^{*}$ \\
H1C & -0.5000 & 0.3688 & 0.7878 & $0.100(8)^{*}$ \\
C2 & $-0.2414(3)$ & $0.3343(2)$ & $0.59501(14)$ & $0.0402(3)$ \\
H2 & -0.4066 & 0.3938 & 0.5861 & $0.048(5)^{*}$ \\
C3 & $-0.0891(3)$ & $0.2941(2)$ & $0.48533(14)$ & $0.0366(3)$ \\
C4 & $0.2333(3)$ & $0.1897(2)$ & $0.33030(14)$ & $0.0368(3)$ \\
C5 & $0.4328(3)$ & $0.1236(2)$ & $0.25071(15)$ & $0.0423(4)$ \\
H5 & 0.5858 & 0.0644 & 0.2813 & $0.049(5)^{*}$ \\
C6 & $0.3978(3)$ & $0.1484(2)$ & $0.12546(16)$ & $0.0470(4)$ \\
H6 & $0.531(3)$ & $0.098(2)$ & $0.0729(18)$ & $0.058(5)^{*}$ \\
C7 & $0.1702(3)$ & $0.2371(2)$ & $0.07882(15)$ & $0.0503(4)$ \\
H7 & 0.1516 & 0.2513 & -0.0065 & $0.055(5)^{*}$ \\
C8 & $-0.0273(3)$ & $0.3040(2)$ & $0.15698(15)$ & $0.0483(4)$ \\
H8 & -0.1790 & 0.3640 & 0.1253 & $0.065(6)^{*}$ \\
C9 & $0.0036(3)$ & $0.2802(2)$ & $0.28572(14)$ & $0.0385(3)$ \\
& & & &
\end{tabular}

Atomic displacement parameters $\left(\AA^{2}\right)$

\begin{tabular}{lllllll}
\hline & $U^{11}$ & $U^{22}$ & $U^{33}$ & $U^{12}$ & $U^{13}$ & $U^{23}$ \\
\hline O1 & $0.0454(7)$ & $0.0711(8)$ & $0.0454(7)$ & $0.0102(6)$ & $-0.0084(5)$ & $-0.0037(6)$ \\
S1 & $0.0344(2)$ & $0.0451(2)$ & $0.0377(2)$ & $0.00088(15)$ & $-0.00453(15)$ & $-0.00450(15)$ \\
N1 & $0.0385(7)$ & $0.0394(7)$ & $0.0402(7)$ & $-0.0011(5)$ & $-0.0012(5)$ & $-0.0029(5)$ \\
N2 & $0.0322(7)$ & $0.0500(7)$ & $0.0399(7)$ & $-0.0034(6)$ & $-0.0031(5)$ & $-0.0038(6)$
\end{tabular}




\begin{tabular}{lllllll} 
C1 & $0.0562(11)$ & $0.0594(11)$ & $0.0411(9)$ & $-0.0060(8)$ & $0.0077(8)$ & $-0.0069(8)$ \\
C2 & $0.0344(8)$ & $0.0425(8)$ & $0.0415(8)$ & $-0.0033(6)$ & $-0.0033(6)$ & $-0.0032(7)$ \\
C3 & $0.0331(7)$ & $0.0355(8)$ & $0.0404(8)$ & $-0.0051(6)$ & $-0.0043(6)$ & $-0.0030(6)$ \\
C4 & $0.0367(8)$ & $0.0362(7)$ & $0.0375(8)$ & $-0.0072(6)$ & $-0.0035(6)$ & $-0.0035(6)$ \\
C5 & $0.0374(8)$ & $0.0431(8)$ & $0.0443(8)$ & $-0.0024(6)$ & $-0.0012(7)$ & $-0.0082(7)$ \\
C6 & $0.0472(9)$ & $0.0492(9)$ & $0.0443(9)$ & $-0.0083(7)$ & $0.0086(7)$ & $-0.0133(7)$ \\
C7 & $0.0555(10)$ & $0.0605(10)$ & $0.0359(8)$ & $-0.0128(8)$ & $-0.0020(7)$ & $-0.0081(8)$ \\
C8 & $0.0420(9)$ & $0.0608(10)$ & $0.0402(9)$ & $-0.0055(7)$ & $-0.0086(7)$ & $-0.0030(8)$ \\
C9 & $0.0369(8)$ & $0.0410(8)$ & $0.0376(8)$ & $-0.0076(6)$ & $-0.0024(6)$ & $-0.0049(6)$ \\
\hline
\end{tabular}

Geometric parameters $\left(\AA,{ }^{o}\right)$

\begin{tabular}{|c|c|c|c|}
\hline $\mathrm{O} 1-\mathrm{N} 1$ & $1.2763(17)$ & $\mathrm{C} 2-\mathrm{C} 3$ & $1.426(2)$ \\
\hline $\mathrm{S} 1-\mathrm{C} 3$ & $1.7456(16)$ & $\mathrm{C} 4-\mathrm{C} 5$ & $1.386(2)$ \\
\hline $\mathrm{S} 1-\mathrm{C} 4$ & $1.7269(17)$ & $\mathrm{C} 4-\mathrm{C} 9$ & $1.393(2)$ \\
\hline $\mathrm{N} 1-\mathrm{C} 1$ & $1.461(2)$ & $\mathrm{C} 5-\mathrm{H} 5$ & 0.9300 \\
\hline $\mathrm{N} 1-\mathrm{C} 2$ & $1.298(2)$ & $\mathrm{C} 5-\mathrm{C} 6$ & $1.371(2)$ \\
\hline $\mathrm{N} 2-\mathrm{C} 3$ & $1.303(2)$ & $\mathrm{C} 6-\mathrm{H} 6$ & $0.934(19)$ \\
\hline $\mathrm{N} 2-\mathrm{C} 9$ & $1.377(2)$ & $\mathrm{C} 6-\mathrm{C} 7$ & $1.389(3)$ \\
\hline $\mathrm{C} 1-\mathrm{H} 1 \mathrm{~A}$ & 0.9600 & $\mathrm{C} 7-\mathrm{H} 7$ & 0.9300 \\
\hline $\mathrm{C} 1-\mathrm{H} 1 \mathrm{~B}$ & 0.9600 & $\mathrm{C} 7-\mathrm{C} 8$ & $1.371(2)$ \\
\hline $\mathrm{C} 1-\mathrm{H} 1 \mathrm{C}$ & 0.9600 & $\mathrm{C} 8-\mathrm{H} 8$ & 0.9300 \\
\hline $\mathrm{C} 2-\mathrm{H} 2$ & 0.9300 & $\mathrm{C} 8-\mathrm{C} 9$ & $1.405(2)$ \\
\hline $\mathrm{C} 4-\mathrm{S} 1-\mathrm{C} 3$ & $88.33(8)$ & $\mathrm{C} 5-\mathrm{C} 4-\mathrm{C} 9$ & $121.64(14)$ \\
\hline $\mathrm{O} 1-\mathrm{N} 1-\mathrm{C} 1$ & $116.50(13)$ & $\mathrm{C} 9-\mathrm{C} 4-\mathrm{S} 1$ & $109.96(12)$ \\
\hline $\mathrm{O} 1-\mathrm{N} 1-\mathrm{C} 2$ & $123.49(13)$ & $\mathrm{C} 4-\mathrm{C} 5-\mathrm{H} 5$ & 121.0 \\
\hline $\mathrm{C} 2-\mathrm{N} 1-\mathrm{C} 1$ & $120.01(13)$ & $\mathrm{C} 6-\mathrm{C} 5-\mathrm{C} 4$ & $117.98(15)$ \\
\hline $\mathrm{C} 3-\mathrm{N} 2-\mathrm{C} 9$ & $109.93(12)$ & $\mathrm{C} 6-\mathrm{C} 5-\mathrm{H} 5$ & 121.0 \\
\hline $\mathrm{N} 1-\mathrm{C} 1-\mathrm{H} 1 \mathrm{~A}$ & 109.5 & $\mathrm{C} 5-\mathrm{C} 6-\mathrm{H} 6$ & $117.3(12)$ \\
\hline $\mathrm{N} 1-\mathrm{C} 1-\mathrm{H} 1 \mathrm{~B}$ & 109.5 & $\mathrm{C} 5-\mathrm{C} 6-\mathrm{C} 7$ & $121.45(15)$ \\
\hline $\mathrm{N} 1-\mathrm{C} 1-\mathrm{H} 1 \mathrm{C}$ & 109.5 & $\mathrm{C} 7-\mathrm{C} 6-\mathrm{H} 6$ & $121.2(12)$ \\
\hline $\mathrm{H} 1 \mathrm{~A}-\mathrm{C} 1-\mathrm{H} 1 \mathrm{~B}$ & 109.5 & $\mathrm{C} 6-\mathrm{C} 7-\mathrm{H} 7$ & 119.6 \\
\hline $\mathrm{H} 1 \mathrm{~A}-\mathrm{C} 1-\mathrm{H} 1 \mathrm{C}$ & 109.5 & $\mathrm{C} 8-\mathrm{C} 7-\mathrm{C} 6$ & $120.85(15)$ \\
\hline $\mathrm{H} 1 \mathrm{~B}-\mathrm{C} 1-\mathrm{H} 1 \mathrm{C}$ & 109.5 & $\mathrm{C} 8-\mathrm{C} 7-\mathrm{H} 7$ & 119.6 \\
\hline $\mathrm{N} 1-\mathrm{C} 2-\mathrm{H} 2$ & 118.2 & $\mathrm{C} 7-\mathrm{C} 8-\mathrm{H} 8$ & 120.6 \\
\hline $\mathrm{N} 1-\mathrm{C} 2-\mathrm{C} 3$ & $123.58(14)$ & $\mathrm{C} 7-\mathrm{C} 8-\mathrm{C} 9$ & $118.84(16)$ \\
\hline $\mathrm{C} 3-\mathrm{C} 2-\mathrm{H} 2$ & 118.2 & $\mathrm{C} 9-\mathrm{C} 8-\mathrm{H} 8$ & 120.6 \\
\hline $\mathrm{N} 2-\mathrm{C} 3-\mathrm{S} 1$ & $116.37(11)$ & $\mathrm{N} 2-\mathrm{C} 9-\mathrm{C} 4$ & $115.40(14)$ \\
\hline $\mathrm{N} 2-\mathrm{C} 3-\mathrm{C} 2$ & $121.29(13)$ & $\mathrm{N} 2-\mathrm{C} 9-\mathrm{C} 8$ & $125.36(14)$ \\
\hline $\mathrm{C} 2-\mathrm{C} 3-\mathrm{S} 1$ & $122.33(11)$ & $\mathrm{C} 4-\mathrm{C} 9-\mathrm{C} 8$ & $119.24(15)$ \\
\hline $\mathrm{C} 5-\mathrm{C} 4-\mathrm{S} 1$ & $128.40(12)$ & & \\
\hline $\mathrm{O} 1-\mathrm{N} 1-\mathrm{C} 2-\mathrm{C} 3$ & $1.7(2)$ & $\mathrm{C} 4-\mathrm{S} 1-\mathrm{C} 3-\mathrm{C} 2$ & $178.11(13)$ \\
\hline $\mathrm{S} 1-\mathrm{C} 4-\mathrm{C} 5-\mathrm{C} 6$ & $179.91(13)$ & $\mathrm{C} 4-\mathrm{C} 5-\mathrm{C} 6-\mathrm{C} 7$ & $0.1(3)$ \\
\hline $\mathrm{S} 1-\mathrm{C} 4-\mathrm{C} 9-\mathrm{N} 2$ & $-0.08(17)$ & $\mathrm{C} 5-\mathrm{C} 4-\mathrm{C} 9-\mathrm{N} 2$ & $-179.70(14)$ \\
\hline $\mathrm{S} 1-\mathrm{C} 4-\mathrm{C} 9-\mathrm{C} 8$ & $-179.91(12)$ & $\mathrm{C} 5-\mathrm{C} 4-\mathrm{C} 9-\mathrm{C} 8$ & $0.5(2)$ \\
\hline $\mathrm{N} 1-\mathrm{C} 2-\mathrm{C} 3-\mathrm{S} 1$ & $1.4(2)$ & $\mathrm{C} 5-\mathrm{C} 6-\mathrm{C} 7-\mathrm{C} 8$ & $0.4(3)$ \\
\hline
\end{tabular}




$\begin{array}{llll}\mathrm{N} 1-\mathrm{C} 2-\mathrm{C} 3-\mathrm{N} 2 & -179.90(14) & \mathrm{C} 6-\mathrm{C} 7-\mathrm{C} 8-\mathrm{C} 9 & -0.4(3) \\ \mathrm{C} 1-\mathrm{N} 1-\mathrm{C} 2-\mathrm{C} 3 & -178.46(14) & \mathrm{C} 7-\mathrm{C} 8-\mathrm{C} 9-\mathrm{N} 2 & -179.78(15) \\ \mathrm{C} 3-\mathrm{S} 1-\mathrm{C} 4-\mathrm{C} 5 & 179.96(15) & \mathrm{C} 7-\mathrm{C} 8-\mathrm{C} 9-\mathrm{C} 4 & 0.0(2) \\ \mathrm{C} 3-\mathrm{S} 1-\mathrm{C} 4-\mathrm{C} 9 & 0.38(11) & \mathrm{C} 9-\mathrm{N} 2-\mathrm{C} 3-\mathrm{S} 1 & 0.71(17) \\ \mathrm{C} 3-\mathrm{N} 2-\mathrm{C} 9-\mathrm{C} 4 & -0.39(19) & \mathrm{C} 9-\mathrm{N} 2-\mathrm{C} 3-\mathrm{C} 2 & -178.07(13) \\ \mathrm{C} 3-\mathrm{N} 2-\mathrm{C} 9-\mathrm{C} 8 & 179.43(15) & \mathrm{C} 9-\mathrm{C} 4-\mathrm{C} 5-\mathrm{C} 6 & -0.5(2) \\ \mathrm{C} 4-\mathrm{S} 1-\mathrm{C} 3-\mathrm{N} 2 & -0.65(12) & & \end{array}$

Hydrogen-bond geometry $\left(\AA,{ }^{\circ}\right)$

\begin{tabular}{lllll}
\hline$D-\mathrm{H} \cdots A$ & $D-\mathrm{H}$ & $\mathrm{H} \cdots A$ & $D \cdots A$ & $D-\mathrm{H} \cdots A$ \\
\hline $\mathrm{C} 5-\mathrm{H} 5 \cdots \mathrm{O} 1^{\mathrm{i}}$ & 0.93 & 2.53 & $3.331(2)$ & 145 \\
\hline
\end{tabular}

Symmetry code: (i) $-x+1,-y,-z+1$. 